Notfall Rettungsmed 2022 $25: 10-18$ https://doi.org/10.1007/s10049-020-00810-8 Online publiziert: 11 . Dezember 2020 (c) Springer Medizin Verlag GmbH, ein Teil von Springer Nature 2020

\section{Zusatzmaterial online}

Die Online-Version dieses Beitrags (https://doi.org/10.1007/s10049020-00810-8) enthält eine Zusammenfassung der gängigsten Merkhilfen (Mnemonics).

Beitrag und Zusatzmaterial stehen Ihnen auf www.springermedizin.de zur Verfügung. Bitte geben Sie dort den Beitragstitel in die Suche ein, das Zusatzmaterial finden Sie beim Beitrag unter „Ergänzende Inhalte“.

\section{Einleitung}

Ziel des vorliegenden Konsensuspapiers ist es, nach Analyse der aktuellen Datenlage konkrete Empfehlungen für den Übergabeprozess in der zentralen

I. Gräff' ${ }^{1}$ M. Pin ${ }^{2} \cdot$ P. Ehlers ${ }^{1} \cdot$ M. Seidel ${ }^{1}$ B. Hossfeld ${ }^{3} \cdot$ M. Dietz-Wittstock ${ }^{4}$. R. Rossi ${ }^{5} \cdot$ A. Gries ${ }^{6} \cdot$ A. Ramshorn-Zimmer ${ }^{6} \cdot$ F. Reifferscheid ${ }^{7} \cdot$ T. Reinhold ${ }^{8}$. H. Band ${ }^{9} \cdot$ K.-H. Kuhl ${ }^{10} \cdot$ M.-K. König ${ }^{11} \cdot$ J. Kasberger ${ }^{12} \cdot$ R. Löb ${ }^{13} \cdot$ R. Krings ${ }^{14}$. S. Schäfer ${ }^{15} \cdot$ I.-M. Wienen ${ }^{16} \cdot$ R. Strametz ${ }^{17} \cdot$ K. Wedler $^{18} \cdot$ C. Mach ${ }^{19} \cdot$ D. Werner ${ }^{20}$. S. Schacher ${ }^{21}$

'Abteilung für Klinische Akut- und Notfallmedizin, Universitätsklinikum Bonn, Bonn, Deutschland; ${ }^{2}$ Zentrale Interdisziplinäre Notaufnahme, Florence-Nightingale-Krankenhaus Düsseldorf, Düsseldorf, Deutschland; ${ }^{3}$ Klinik für Anästhesiologie, Intensivmedizin, Notfallmedizin u. Schmerztherapie, Bundeswehrkrankenhaus Ulm, Ulm, Deutschland; ${ }^{4}$ Zentrale Notaufnahme, Diakonissenkrankenhaus Flensburg, Flensburg, Deutschland; ${ }^{5}$ Notarztdienst Landkreis Schwäbisch Hall, Schwäbisch Hall, Deutschland; ${ }^{6}$ Zentrale Notfallaufnahme, Universitätsklinikum Leipzig, Leipzig, Deutschland; ${ }^{7}$ Klinik für Anästhesiologie und Operative Intensivmedizin, Universitätsklinikum Schleswig-Holstein, Kiel, Deutschland; ${ }^{8}$ Notaufnahme, Klinik Oranienburg Oberhavel-Kliniken $\mathrm{GmbH}$, Oranienburg, Deutschland; ${ }^{9}$ Verband der Feuerwehren in Nordrhein-Westfalen, Wuppertal, Deutschland; ${ }^{10}$ Arbeitsgemeinschaft der Leiter der Berufsfeuerwehren, Bonn, Deutschland; "Deutscher Berufsverband Rettungsdienst Lübeck, Lübeck, Deutschland; ${ }^{12}$ Deutsches Rotes Kreuz, Landesverband Nordrhein e. V., Düsseldorf, Deutschland; ${ }^{13}$ Klinik für Anästhesiologie, Intensiv-, Notfall- und Schmerztherapie, St. Barbara-Klinik Hamm, Hamm, Deutschland; ${ }^{14}$ Johanniter-Unfall-Hilfe, Landesverband Nordrhein-Westfalen, Köln, Deutschland; ${ }^{15}$ Arbeiter-Samariter-Bund, Köln, Deutschland; ${ }^{16}$ Falck Notfallrettung und Krankentransport, Region Nordrhein-Westfalen, Köln, Deutschland; ${ }^{17}$ Hochschule Rhein Main, Wiesbaden, Deutschland; ${ }^{18} \mathrm{Christliche} \mathrm{Akademie} \mathrm{für} \mathrm{Gesundheits-} \mathrm{und} \mathrm{Pflegeberufe,} \mathrm{Halle,} \mathrm{Deutschland;}{ }^{19}$ Zentrale Notaufnahme, Uniklinik RWTH Aachen, Aachen, Deutschland; ${ }^{20}$ ADAC Luftrettung München, München, Deutschland;

${ }^{21}$ Zentrale Notaufnahme, Evangelisches Krankenhaus Köln Kalk, Köln, Deutschland

\title{
Empfehlungen zum strukturierten Übergabeprozess in der zentralen Notaufnahme
}

\section{Konsensuspapier von DGINA, DIVI, BAND, BV-AELRD, VDF, AGBF, DBRD, DRK, MHD, JUH, ASB, FALCK, APS, ABNP, DRF, ADAC}

Notaufnahme hinsichtlich Inhalten und Struktur zu machen. Mit der Übergabe vom Rettungsdienst an die zentrale Notaufnahme beginnt für eine Vielzahl von Notfallpatienten der klinische Behandlungsprozess, welcher den im Folgenden beschriebenen Rahmenbedingungen unterliegt. Daher liegt der Hauptfokus des Konsensuspapiers auf dieser Schnitt-/Nahtstelle. Darüber hinaus lassen sich Kernbestandteile der Übergabe bzw. des Übergabeprozesses und somit die ausgesprochenen Empfehlungen auch auf andere Schnittstellen, wie z. B. die innerklinischen, übertragen.

Die Übergabe in der Medizin ist definiert als die Übertragung der Verantwortlichkeit und Zuständigkeit für einige oder alle Aspekte der Versorgung eines oder mehrerer Patienten an eine andere Person oder Berufsgruppe für vorübergehende oder längere Zeit $[1,2]$.

Als Bestandteil eines Gesamtbehandlungsprozesses schließt sie einerseits die präklinische Versorgung ab und muss andererseits an der Nahtstelle zur zentralen Notaufnahme (ZNA) durch die Weitergabe der bisherigen Behandlungs- und Patienteninformationen die Kontinuität und Sicherheit im Gesamtbehandlungsprozess gewährleisten. Nach der Übergabe beginnt der innerklinische Behandlungsprozess.

In der Regel existiert für die mündliche/schriftliche Übergabe und die Übermittlung patientenrelevanter Informationen nur eine einmalige Möglichkeit. Der Übergabe kommt somit eine immense Bedeutung zu ([3]; - Abb. 1). 


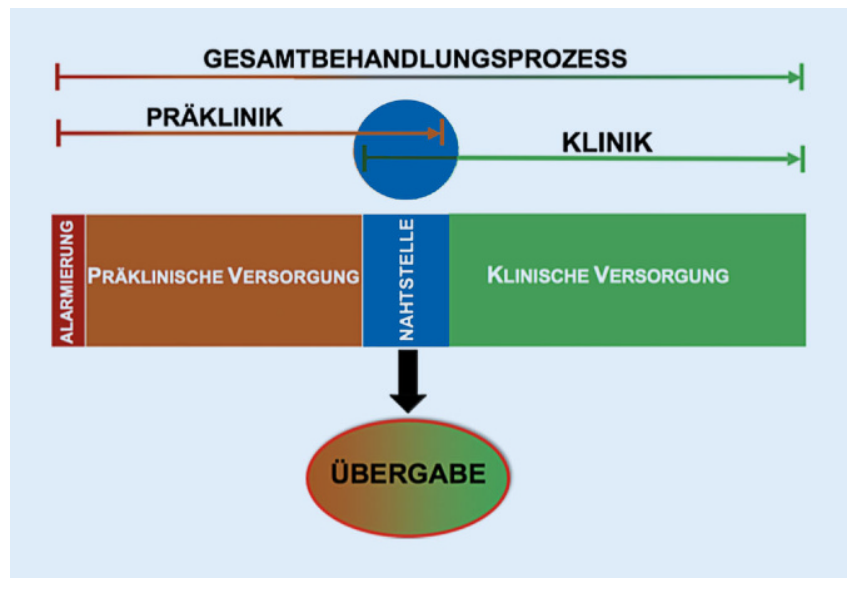

Abb. $1<$ Darstellung des Gesamtbehandlungsprozesses mit der Übergabe als Nahtstelle der präklinischen Versorgung zur klinischen Versorgung
Speziell in zentralen Notaufnahmen werden Übergaben als komplex beschrieben und sind mit einem erhöhten Fehlerrisiko aufgrund des hohen Stressund Zeitdrucks bei der Versorgung der Patienten behaftet $[4,5]$. Gleichzeitig kommt erschwerend das differente Kompetenzniveau der beteiligten Berufsgruppen hinzu. Nahezu täglich erleben Mitarbeiter von Notaufnahmen eine rasche Patientenfluktuation, hohe Patientenzahlen sowie Inkonsistenzen und Unvorhersehbarkeiten im Arbeitsalltag $[3,6,7]$. Im Gegensatz zu Schichtübergaben auf den Stationen ist die Übergabe von der präklinischen Versorgung an die ZNA immer auch eine interprofessionelle Übergabe, an der mindestens zwei Berufsgruppen beteiligt sind, nämlich Rettungsdienst und Pflege, sowie häu-

Dieses Konsensuspapier wurde initiiert durch: Deutsche Gesellschaft für interdisziplinäre Notfall- und Akutmedizin e.V. (DGINA); Deutsche Interdisziplinäre Vereinigung für Intensiv- und Notfallmedizin e.V. (DIVI); unter Mitwirkung von: ADAC Luftrettung gGmbH; Aktionsbündnis Notfallpflege (ABNP); Aktionsbündnis Patientensicherheit e.V. (APS); Arbeiter-Samariter-Bund e. V. (ASB); Arbeitsgemeinschaft der Leiter der Berufsfeuerwehren (AGBF); Bundesvereinigung der Arbeitsgemeinschaften der Notärzte Deutschlands e.V. (BAND); Bundesverband der Ärztlichen Leitung Rettungsdienst Deutschland e.V. (BV-ÄLRD); Deutscher Berufsverband Rettungsdienst e.V. (DBRD); Deutsches Rotes Kreuz Landesverband Nordrhein e.V. (DRK); DRF Stiftung Luftrettung gemeinnützige AG; Falck Notfallrettung und Krankentransport GmbH; Malteser Hilfsdienst Bundeszentrale; Johanniter-Unfall-Hilfe e.V. (JUH); Verband der Feuerwehren in Nordrhein-Westfalen e.V.(VDF) fig auch noch eine dritte Berufsgruppe, die Ärzte. Im Zuge der zunehmenden telemedizinischen Unterstützung von Notfallsanitätern wird es vermehrt Übergaben geben, bei denen im Vorfeld ein Arzt (Telenotarzt) involviert war, welcher dann aber persönlich bei der Übergabe nicht anwesend ist. Die verschiedenen Erwartungen und fachlichen Voraussetzungen der einzelnen Berufsgruppen bei der Übergabe können zu Missverständnissen und Unzufriedenheit führen $[4,8]$. Außerdem konnten Untersuchungen der Australian Commission on Safety and Quality in Health Care (ACSQHC) zeigen, dass gerade bei Vorliegen komplexer Patientenprobleme die Übergabequalität abnimmt [9].

\section{Studienlage zur Übergabe}

Die häufigste Ursache für sog. SentinelEreignisse, die der Joint Commission on Accreditation of Healthcare Organizations (JCAHO) in den Vereinigten Staaten von Amerika zwischen 1995 und 2006 gemeldet wurden, war inadäquate Kommunikation [3]. Daraus folgend ist seit 2006 in den USA das Thema Patientenübergabe in den Fokus gerückt und als Konsequenz wurde die Einführung standardisierter Patientenübergaben zur Erhöhung der Patientensicherheit als Ziel formuliert [10].

In einer longitudinalen Untersuchung wurde gezeigt, dass Kommunikationsfehler in US-amerikanischen Krankenhäusern und Arztpraxen für 30\% aller Behandlungsfehlervorwürfe verantwortlich sind. Dies führte über einen Zeitraum von 5 Jahren zu 1744 Todesfällen und Kosten in Höhe von 1,7 Mrd. US-Dollar [11]. Daten aus Australien zeigen, das von 25.000 bis 30.000 unerwünschten, potenziell vermeidbaren Ereignissen, die $\mathrm{zu}$ einer dauerhaften Schädigung führten, $11 \%$ auf Kommunikationsprobleme zurückzuführen waren [12]. Scott et al. haben in ihrer Studie untersucht, welche mündlich übermittelten Informationen unmittelbar nach der Übergabe beim aufnehmenden Personal noch abrufbar waren. Insgesamt erinnerten sich die Ärzte genau an $36 \%$ des Inhalts der mündlichen Übergabe. Informationen von weniger schwer verletzten Notfallpatienten konnten besser wiedergegeben werden als von schwer verletzten Notfallpatienten [13]. Bogenstätter et al. fanden in ihrer Simulationsstudie im Setting einer Notfallambulanz heraus, dass Übergabeinformationen nur teilweise zuverlässig sind [14]. Evans et al. haben in ihrer Untersuchung ebenfalls herausgestellt, dass Informationen bei der Übergabe von Traumapatienten fehlten bzw. falsch übermittelt wurden [15]. Theobald et al. haben den Übergabeprozess bei Interhospitaltransporten untersucht und konnten zeigen, dass unter Anwendung eines standardisierten Übergabeprotokolls die Krankenhaussterblichkeit signifikant gesenkt werden konnte [16].

Ein in der Literatur beschriebenes Instrument zur Verbesserung des Übergabeprozesses ist das sog. Targeted Solutions Tool $\left(\mathrm{TST}^{\circledR}\right)$, hierbei handelt es sich um eine innovative Anwendung zur Fehlerdetektion im Rahmen der Übergabe [10]. In einer Studie im Bartlett Regional Hospital in Juneau, Alaska, konnte gezeigt werden, dass mit der Anwendung des TST $^{\circledR}$ vier Hauptfaktoren für $69,8 \%$ der fehlerhaften Übergaben verantwortlich waren. Hierbei handelt es sich um ungenaue/unvollständige Informationen, ineffektive Methoden (schriftlich/mündlich), keine standardisierten Verfahren für eine effektive Übergabe und mangelndes Wissen bei der übergebenden Person [17].

Keebler et al. griffen mit ihrer Untersuchung im Jahr 2017 die von der JCAHO im Jahr 2007 geforderte Standardisierung der Übergabe auf und stellen fest, dass die Standardisierung in al- 
len Studien unterschiedlich ist und somit eine Vergleichbarkeit nur eingeschränkt möglich ist [18].

Diese reicht von der Einführung einer Merkhilfe (Mnemonic wie z.B. ISBAR, IPASS), computergestützten Übergabeprogrammen sowie Protokollen/Übergabealgorithmen bis hin zu Etablierung von Checklisten.

Daraus folgerten Keebler et al., dass Protokolle prinzipiell funktionieren. Sie objektivieren die Übergabe und geben dem Anwender eine Orientierung, welche Informationen übermittelt werden sollen. Insgesamt führen sie dazu, dass die Beteiligten der Übergabe in einem gemeinsamen Gedankenmodell („shared mental model“) agieren. Dementsprechend haben alle "Sender" das gleiche Verständnis, welche Informationen sie übermitteln sollten, und alle „Empfänger" wissen, welche Informationen sie benötigen bzw. erwarten können. Diese strukturierte Kommunikation verbessert im klinischen Versorgungsspektrum das Outcome und beeinflusst darüber hinaus die Ergebnisse auf organisatorischer Ebene. Beispielweise ist hier der Anspruch des Advanced Trauma Life Support $\left(\right.$ ATLS $\left.^{\circledR}\right)$ zu nennen, bei dem eine „gemeinsame Sprache“ („commmon language of trauma") gelehrt wird [19].

\section{Bisherige Konzepte und Leitlinien}

In Bezug auf eine Merkhilfe empfiehlt die WHO seit 2007 die Nutzung des SBARSchemas (Situation, Backround, Assessment, Recommendation) bei der Übergabe von Patienten zur Verbesserung der Patientensicherheit [20].

Im Jahr 2008 hat die Weltgesundheitsorganisation (WHO) die Entwicklung von Standard Operating Procedures (SOP) in der Kommunikation als eine der fünf Prioritäten im Bereich Patientensicherheit für Länder der ersten Welt formuliert [21].

In Deutschland gibt es bisher keine Initiative, die auf nationaler Ebene versucht, den Übergabeprozess zu verbessern und als gesundheitspolitisches Ziel zu definieren.

Notfall Rettungsmed 2022 $25: 10-18$ https://doi.org/10.1007/s10049-020-00810-8

(c) Springer Medizin Verlag GmbH, ein Teil von Springer Nature 2020

I. Gräff · M. Pin · P. Ehlers · M. Seidel • B. Hossfeld • M. Dietz-Wittstock · R. Rossi · A. Gries · A. Ramshorn-Zimmer · F. Reifferscheid · T. Reinhold · H. Band · K.-H. Kuhl · M.-K. König · J. Kasberger · R. Löb · R. Krings · S. Schäfer · I.-M. Wienen · R. Strametz · K. Wedler · C. Mach · D. Werner $\cdot$ S. Schacher

\section{Empfehlungen zum strukturierten Übergabeprozess in der zentralen Notaufnahme. Konsensuspapier von DGINA, DIVI, BAND, BV-AELRD, VDF, AGBF, DBRD, DRK, MHD, JUH, ASB, FALCK, APS, ABNP, DRF, ADAC}

\section{Zusammenfassung}

Der Übergabe als Übertragung der Verantwortlichkeit und Zuständigkeit für die weitere Versorgung des Patienten kommt eine besondere Bedeutung im Behandlungsprozess und für die Patientensicherheit zu. Dies gilt umso mehr für die Übergabe des Rettungsdiensts an die zentrale Notaufnahme in ihrem komplexen und stressbehafteten Umfeld. Dieser Prozess ist auf eine in allen Aspekten funktionierende Kommunikation angewiesen, um unerwünschte Ereignisse für den Patienten zu vermeiden und eine optimale Weiterversorgung zu gewährleisten. Bisher existieren in Deutschland nur in einzelnen Bereichen überhaupt Vorgaben und Konzepte für eine strukturierte Übergabe. Im vorliegenden Konsensuspapier werden fünfzehn Empfehlungen für einen standardisierten Übergabeprozess des Notfallpatienten vorgestellt, die durch die Fachgesellschaften der an der Übergabe beteiligten Berufsgruppen (Notfallmediziner, Notfallpflege, Rettungsdienstorganisationen) sowie das Bündnis für Patientensicherheit als Vertreter der Patienten erarbeitet wurden.

Schlüsselwörter

Übergabe · Notfallmedizin · Kommunikation . Patientensicherheit $\cdot$ Merkhilfe

\section{Recommendations for a structured handover process in the emergency department. Consensus paper from DGINA, DIVI, BAND, BV-AELRD, VDF, AGBF, DBRD, DRK, MHD, JUH, ASB, FALCK, APS, ABNP, DRF, ADAC}

\section{Abstract}

Handover as a transfer of responsibility and accountability of further patient care is an important moment in the treatment process and in regard of patient safety. This applies especially to handovers between paramedics and the emergency department staff, in a complex and stressful environment. This process relies on communication that has to work in all regards to avoid adverse events for the patient and to ensure optimal continuity of care. At the moment, there are very few guidelines and concepts for a structured handover in Germany. The present consensus paper introduces fifteen recommendations for a standardized handover process of the emergency patient, which were developed by the professional associations, representing the professions involved in the handover (emergency physicians, emergency nurses, paramedics and ambulance services) as well as the German Coalition for Patient Safety as a representative of the patients.

Keywords Handover - Emergency medicine . Communication - Patient safety $\cdot$ Mnemonic
Es existieren lediglich singuläre Publikationen und Engagements zum Thema Übergabe.

Der Deutsche Berufsverband Rettungsdienst (DBRD) hat bereits im Jahr 2007 ein Positionspapier zur Voranmeldung und Übergabe von Patienten an der Schnittstelle Rettungsdienst/Klinik veröffentlicht [22].
Die Deutsche Gesellschaft für Anästhesiologie und Intensivmedizin (DGAI) hat auf Basis eines Beschlusses des engeren Präsidiums im Jahr 2016 das SBARKonzept (situation, backround, assessment, recommendation) für die strukturierte Übergabe in der perioperativen Phase empfohlen [23].

Darüber hinaus werden in Deutschland Ausbildungskonzepte angeboten, 
Tab. 1 Auflistung der Aufgaben und Anforderungen einer Merkhilfe

\begin{tabular}{|c|c|}
\hline Aufgaben einer Übergabemerkhilfe & Anforderungen an eine Übergabemerkhilfe \\
\hline $\begin{array}{l}\rightarrow \text { Erhöht die Sicherheit für den Patienten } \\
\text { und alle Beteiligten }\end{array}$ & $\rightarrow$ Anwendbar durch alle Berufsgruppen \\
\hline$\rightarrow$ Unterstützt systematisches Arbeiten & $\rightarrow$ Einsatz für Akut- und Notfallpatienten \\
\hline $\begin{array}{l}\rightarrow \text { Gibt der Übergabe einen logischen Auf- } \\
\text { bau }\end{array}$ & $\begin{array}{l}\rightarrow \text { Abdeckung für verletzte und erkrankte Patien- } \\
\text { ten aller Fachrichtungen }\end{array}$ \\
\hline $\begin{array}{l}\rightarrow \text { Erleichtert die konzentrierte Weitergabe } \\
\text { aller wichtigen Informationen }\end{array}$ & $\begin{array}{l}\rightarrow \text { Einsetzbar für Patienten aller Schweregrade } \\
\text { und Altersgruppen }\end{array}$ \\
\hline $\begin{array}{l}\rightarrow \text { Verhindert Informationsverlust und Miss- } \\
\text { verständnisse }\end{array}$ & $\begin{array}{l}\rightarrow \text { Erlaubt Integration gängiger Schemata und } \\
\text { Scores (z. B. cABCDE, NRS, SAMPLER) }\end{array}$ \\
\hline $\begin{array}{l}\rightarrow \text { Erhöht die Effizienz des Übergabevor- } \\
\text { gangs }\end{array}$ & $\begin{array}{l}\rightarrow \text { Unabhängig vom Ausbildungsstand erlern- } \\
\text { und anwendbar }\end{array}$ \\
\hline $\begin{array}{l}\rightarrow \text { Verbessert die Zusammenarbeit zwischen } \\
\text { patientenbetreuenden Teams }\end{array}$ & $\rightarrow$ Zeitsparend und prioritätenorientiert \\
\hline $\begin{array}{l}\rightarrow \text { Fördert den Teamgeist und erhöht die } \\
\text { Mitarbeiterzufriedenheiten }\end{array}$ & $\rightarrow$ Unempfindlich gegenüber Störeinflüssen \\
\hline $\begin{array}{l}\rightarrow \text { Soll eine strukturierte Dokumentation } \\
\text { ermöglichen }\end{array}$ & $\begin{array}{l}\rightarrow \text { Situativ anpassbar an alle Schnittstellen in } \\
\text { Präklinik und Klinik }\end{array}$ \\
\hline
\end{tabular}

die standardisierte diagnostische und therapeutische Handlungsabläufe für die präklinische oder die frühe innerklinische Erstversorgung von schwer verletzten bzw. schwer erkrankten Patienten definieren. Im European Trauma Course $\left(\mathrm{ETC}^{\circledR}\right)$ wird beispielsweise das AT-MIST-Schema (age, time- mechanism, injury, signs, treatment) zur fokussierten Traumaübergabe im Schockraum gelehrt. Zur strukturierten und schnellen Erfassung von bedrohlichen Verletzungen und Störungen der Vitalfunktionen wird der $<\mathrm{C}>\mathrm{ABCDE}$-Algorithmus (airway, breathing, circulation, disability, environment) herangezogen, zur strukturierten Anamneseerhebung das SAMPLER-Schema (symptoms, allergies, medication, past medical history, last oral intake, events, risk factors) [24].

Vergleicht man diese mit der aktuellen Literatur so muss konstatiert werden, dass es sich bei beiden Angaben nicht um ein Übergabeschema für die ZNA im klassischen Sinne handelt [25].

In der aktuellen S3-Leitlinie zur Polytrauma-/Schwerverletztenbehandlung wird als Good Practice Point (GPP) aufgeführt, dass zur Vermeidung von Schnittstellenproblemen bei der Übergabe bzw. Übernahme von schwer verletzten Patienten geeignete und standardisierte Kommunikationsmethoden verwendet werden sollen [26].

\section{Die Merkhilfe als Kernbestand- teil der Übergabe}

In der Literatur sind inzwischen eine Vielzahl von Merkhilfen (Mnemonics) zur Standardisierung der mündlichen Übergabe publiziert worden ([25, 27]; eine Zusammenstellung finden Sie im Online-Zusatzmaterial, siehe Hinweisbox am Anfang des Beitrags).

Mnemonics sind Gedächtnishilfen, die eingängig sein sollen und ein Akronym mit einem Prozess verknüpfen. In - Tab. 1 sind die Aufgaben und Anforderungen einer Übergabemerkhilfe aufgelistet.

\section{Umfassend - sicher - einfach - robust}

Die Merkhilfe sollte als „Skelett“ für eine umfassende, zeitlich begrenzte Übergabe angesehen werden. Zum gegenwärtigen Zeitpunkt lässt die Analyse der Datenlage allerdings keinen Rückschluss $\mathrm{zu}$, welches für die Übergabe die $\mathrm{zu}$ favorisierende Merkhilfe ist. Im direkten Vergleich zeigt sich, dass sie aus unterschiedlichen Motivationen heraus entwickelt wurden und deshalb alle einen unterschiedlichen Fokus haben. Nichtsdestotrotz lassen sich einige Anforderungen an eine geeignete Merkhilfe für die Übergabe in der ZNA definieren [25]. Die Merkhilfe muss eingängig sein und dem Anwender eine gute Gedächtnisstütze für die zu übergebenden
Inhalte geben. Der Aufbau der Merkhilfe sollte in sich logisch sein und eine praxisbezogene Reihenfolge mit sich bringen. Komplexe Patienten sollten in der Merkhilfe genauso abgebildet sein wie weniger kritische Notfallpatienten. Angaben zur medizinischen Situation bzw. zum Unfallmechanismus, der präklinische Untersuchungsbefund und das führende Problem, im bewährten $<\mathrm{C}>\mathrm{ABCDE}$-Algorithmus, müssen in der Merkhilfe enthalten sein. Abschließen sollte die Merkhilfe inhaltlich mit den präklinischen Notfallmaßnahmen und mit relevanten Informationen zur allgemeinen Krankengeschichte/zu soziodemografischen Daten sowie Risikofaktoren und Allergien [28].

Im Online-Zusatzmaterial dieses Beitrags finden Sie eine Übersicht der am häufigsten in der Literatur beschrieben Merkhilfen.

\section{Empfehlungen zur Übergabe}

Gibson et al. beschreiben in ihrer Arbeit 4 Phasen der Übergabe [29]:

- Vorbereitung

- Zusammenkommen - hier die bewusste Unterbrechung der Arbeitsabläufe!

- Dialog - Austausch

- Zusammenfassung

Betrachtet man den Übergabeprozess aus Crew-Ressource-Management(CRM)-

Gesichtspunkten, so wird deutlich, dass CRM-Aspekte wie Kooperation, situative Aufmerksamkeit, Führungsverhalten, Entscheidungsfindung sowie sichere und effektive Kommunikation in der Übergabe eine wesentliche Rolle spielen [30].

\section{Empfehlung 1}

Crew-Ressource-Management-Prinzipien sollen immer die Basis des Übergabeprozesses bilden.

Im Sinne eines gestörten „SenderEmpfänger-Modells“ besteht das Risiko einer Schädigung des Patienten, wenn der „Empfänger“ Informationen erhält, die ungenau oder unvollständig sind. Darüber hinaus führen eine unstrukturierte Informationsweitergabe, falsche 


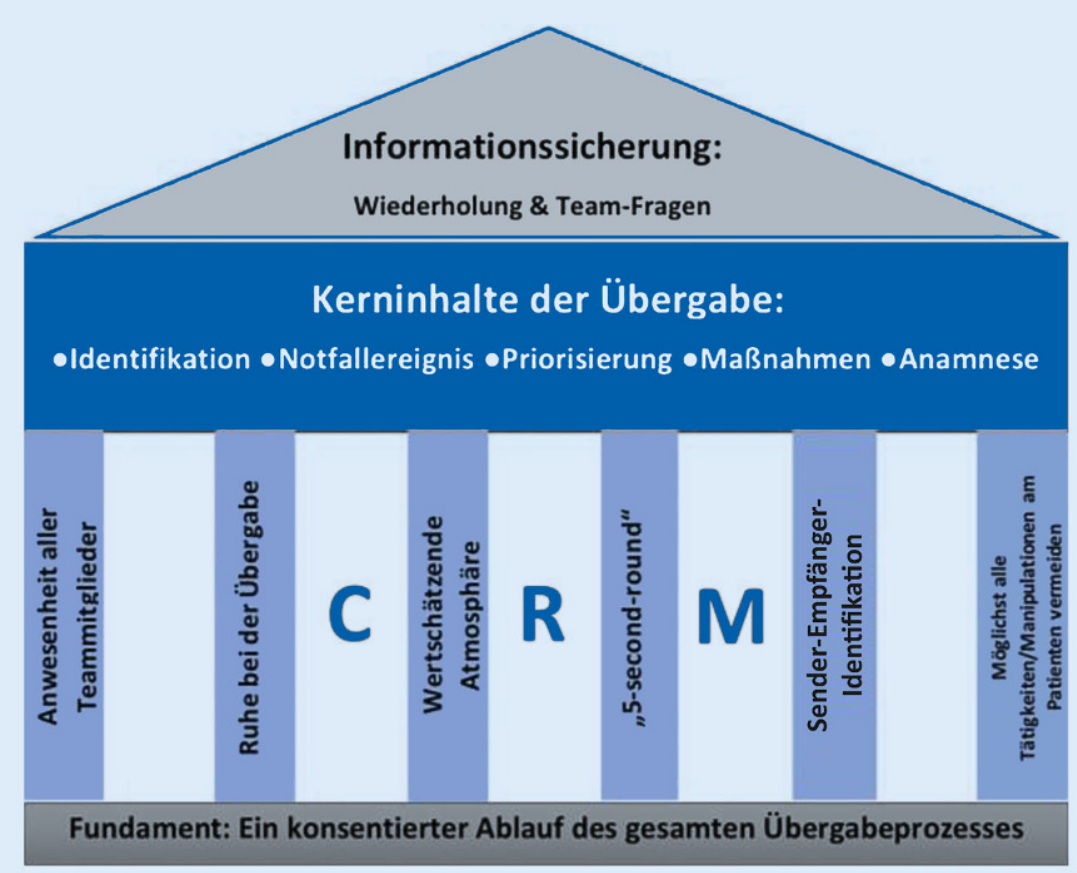

Abb. 2 \ Schematische Darstellung der mündlichen Übergabe in der ZNA. Basis ist ein konsentierter Übergabeprozess. Kernbestandteil ist die Merkhilfe, welche durch CRM-Prinzipien "getragen" wird. Identifikation beinhaltet Alter, Geschlecht und Name. Notfallereignis impliziert die 4 „W-Fragen“: was (Leitsymptom/Verdachtsdiagnose), wie (Beschreibung des Ereignisses), wo (Ort) und wann (Zeit-

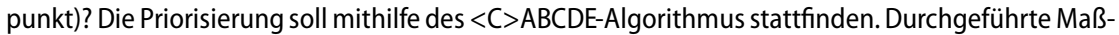
nahmen werden inklusive deren Wirkung übergeben. Die Übergabe der Anamnese des Patienten sollte sich am sog. SAMPLER-Schema orientieren. Am Ende der Übergabe steht die Kurzwiederholung durch den „Empfänger", und gezielte Rückfragen sind möglich

Interpretation von vermittelten Inhalten oder nichtrelevante Informationen $\mathrm{zu}$ einer zusätzlichen Patientengefährdung $[3,31]$.

Verläuft die Übergabe nicht standardisiert und ohne Konzentration auf die Inhalte der Informationsweitergabe, verliert sie deutlich an Wirkung. Merkhilfen als Kernbestandteil der mündlichen Übergabe sollen genau diesen negativen Einflussgrößen entgegenwirken ([25]; - Abb. 2).

\section{Empfehlung 2}

\section{Die Übergabe kritischer Inhalte soll standardisiert, unter Zuhilfenahme einer bundesweit einheitlich konsen- tierten Merkhilfe, stattfinden.}

Die Autoren des vorliegenden Konsensuspapiers validieren derzeit mit einem zweistufigen Verfahren eine neu entwickelte Merkhilfe, die die Empfehlungen des Konsenspapiers erfüllt und hier- bei CRM-Prinzipien fest integriert hat. Diese Ergebnisse werden getrennt publiziert und sollen die Grundlage für eine bundesweit einheitlich konsentierte Merkhilfe liefern.

\section{Zentrale und standardisierte Anmeldung in der ZNA}

Eine Vorabinformation über die Zuführung eines Notfallpatienten mit erhöhten medizinischen, logistischen, infrastrukturellen Anforderungen, unabhängig davon, ob traumatologisch oder nichttraumatologisch, sollte in der zentralen Notaufnahme erfolgen. Die ZNA braucht zur Vorbereitung essenzielle Informationen über den Patienten, daher ist es unabdingbar, die Anmeldung standardisiert durchzuführen [22].

Das kann entweder telefonisch über ein sog. "Rotes Telefon“ erfolgen oder wie vielerorts in Deutschland über eine webbasierte Anwendung (z. B. „IVENA eHealth“, „Rescue Track“ etc.).
Eine einheitliche, strukturierte Anmeldung für kritisch kranke Patienten minimiert Informationsverluste und ermöglicht den aufnehmenden Kliniken eine zeitgerechte und patientenorientierte Vorbereitung zur nahtlosen (Weiter-)Versorgung [32].

Eine enge Absprache und verbindlich strukturierte Zusammenarbeit mit dem örtlichen Rettungsdienst ist daher unabdingbar.

\section{Empfehlung 3}

\section{Die Anmeldung eines Notfallpatienten durch den Rettungsdienst soll standar- disiert in der ZNA erfolgen.}

\section{Vorbereitung der ZNA}

Sharon Ryan und Mitarbeiter haben in ihrer Untersuchung nachweisen können, dass das Outcome der Patienten durch ein adäquates Teambriefing positiv beeinflusst wird. Je besser das Team auf die anstehende Herausforderung vorbereitet ist, desto strukturierter kann die Übergabe und somit auch die Versorgung stattfinden [33]. Das CRM-Prinzip „Antizipiere und plane voraus" hat gerade beim Übergabeprozess eine enorme Bedeutung. Die interdisziplinäre Aufgabenverteilung und der Ablauf sollten daher sowohl logistisch wie auch medizinisch im Vorfeld eindeutig geregelt sein und regelmäßig geschult werden [30].

\section{Empfehlung 4}

Vor Ankunft von kritisch kranken Notfallpatienten soll ein Teambriefing mit allen Beteiligten der Notfallversorgung stattfinden.

Es empfiehlt sich, die Informationen, welche vorab im Rahmen der Anmeldung übermittelt worden sind, plakativ im Schockraum an eine Anzeigetafel (z. B. ein Whiteboard) zu schreiben. Idealerweise orientiert sich die Struktur der Anzeigetafel an der vom Rettungsdienst angewendeten Merkhilfe. Dies hat mehrere Vorteile. Zum einen können während der mündlichen Übergabe noch fehlende Informationen ergänzt werden, zum anderen können die dann verfügbaren Informationen zur Rekapitulation heran- 
gezogen werden. Zusätzlich haben später hinzugezogene Teammitglieder direkt einen Überblick über alle initialen Informationen.

\section{Empfehlung 5}

Informationen aus der Anmeldung und der Übergabe sollen für alle sichtbar im Schockraum zur Verfügung stehen.

\section{„Face-to-face-Übergabe" und "hands off"}

Bundeseinheitlich wird nach Empfehlung der Deutschen Interdisziplinären Vereinigung für Intensiv- und Notfallmedizin (DIVI) im Rettungsdienst der Datensatz zur Dokumentation rettungsdienstlicher/notärztlicher Einsätze als sog. DIVI-Protokoll genutzt [34]. Dieses Protokoll ermöglicht eine umfassende, detaillierte und systematische Dokumentation des rettungsdienstlichen Einsatzes, kann allerdings die mündliche Übergabe in der ZNA nicht ersetzen. Solet et al. schlussfolgern in ihrer Arbeit, das eine eindeutige Kommunikation von Angesicht zu Angesicht der beste Weg ist, eine effektive Übergabe von Krankenhauspatienten zu gewährleisten [35]. Ausschließlich elektronische Übergaben oder in Papierform sollen vermieden werden, sehr wohl kann die Übergabe mit visueller Unterstützung (z.B. Pocketcard) erfolgen.

Darüber hinaus sollte die Übergabe in einer ruhigen, freundlichen und wertschätzenden Umgebung am Patienten stattfinden.

\section{Empfehlung 6}

Die Übergabe soll interaktiv zwischen "Sender" und „Empfänger" ausgerichtet sein und in einer freundlichen, wertschätzenden Atmosphäre stattfinden.

Gleichzeitig spiegelt diese Fokussierung während der Übergabe eine besondere Art des Respekts gegenüber dem Empfänger der Nachricht wider. Von „Angesicht zu Angesicht“ ist bei der Übergabe zwischen zwei Einzelpersonen wörtlich zu nehmen [36].
Bei der Übergabe von kritisch kranken Patienten im Schockraum sollte darauf geachtet werden, dass die Übergabeinformationen an alle Teammitglieder gerichtet werden.

Auf der Seite des aufnehmenden Teams soll ein klarer Ansprechpartner kenntlich gemacht werden (Teamleader). Vor Beginn der Übergabe sollte unbedingt eine kurze klinische Prüfung stattfinden, („5-second round“), um zu gewährleisten, dass der Patient durch die Phase der Übergabe nicht zusätzlich gefährdet wird. Bei Patientenzuständen (z.B. laufende cardiopulmonale Reanimation CPR), die keine Unterbrechung zur Übergabe zulassen, ist eine sofortige Umlagerung bei Eintreffen des Rettungsdiensts anzustreben. Hier kann die Übergabe trotzdem primär an den Teamleader stattfinden, der dann sekundär zeitnah die Information $\mathrm{zu}$ einem geeigneten Zeitpunkt ins Team trägt [37].

\section{Empfehlung 7}

Die Übergabe soll bei Anwesenheit aller an der Versorgung beteiligten Teammitglieder erfolgen.

Idealerweise findet die Übergabe in einem Arbeitsbereich oder einem Umfeld statt, das den Austausch von Informationen über einen Patienten fördert, wie z.B. einer Zone, die frei von nichtnotfallbedingten Unterbrechungen ist. Untersuchungen mit Pflegekräften zeigen, dass eine laute Geräuschkulisse während der Übergabe nachteilige Auswirkungen insbesondere auf die mnestischen Fähigkeiten hat [38]. Darüber hinaus reduziert Lärm die Speicherung komplexer Sachverhalte und verursacht Stress, welcher mit einem erhöhten Fehler- und Personalausfallrisiko einhergeht [39].

Der Teamleader sorgt für die notwendige Ruhe und sorgt mit der Initiierung der Übergabe dafür, dass alle Teammitglieder zuhören und keine Manipulationen am Patienten vorgenommen werden. Untersuchungen zeigen, dass gerade diese Konzentration und Fokussierung die Übergabequalität verbessert [13].

\section{Empfehlung 8}

Die Übergabe soll bei minimaler Geräuschkulisse stattfinden. Manipulationen während der Übergabe sollten unterlassen werden.

In anderen Bereichen wie z. B. im OP hat das sog. „Time-out" signifikant zu einer Verringerung der Fehler und zur Senkung der Sterblichkeit geführt [40].

\section{Wiederholung und Fragen}

Am Ende der Übergabe sollten im Sinne einer geschlossenen Kommunikation die essenziellen Übergabeinformationen durch den „Empfänger“ rekapituliert werden. Nur so ist sichergestellt, basierend auf dem Sender-EmpfängerModell, dass das Gesagte auch inhaltlich richtig verstanden wurde.

\section{Empfehlung 9}

\section{Am Ende der Übergabe sollten die In- formationen kurz laut und verständlich zusammengefasst werden.}

Am Ende der Übergabe sind Fragen zu übergaberelevanten Aspekten ausdrücklich von jedem Teammitglied erwünscht. Insgesamt sollte die Übergabe nicht länger als zwei Minuten dauern. Keiner kennt den Patienten zum Zeitpunkt der Übergabe so gut wie die Besatzung des zuführenden Rettungsmittels. Unabhängig davon, ob ärztlich besetzt oder nicht, sollte im Rahmen der Übergabe die Möglichkeit bestehen, besondere Hinweise aussprechen zu dürfen. Basis für diese Empfehlung wird somit die präklinische Betreuungszeit bzw. die Arbeitshypothese/Verdachtsdiagnose des Rettungsdiensts sein.

Im Kontext der ausgesprochenen Empfehlung kann es zum sog. „Framing-Effekt" kommen, dieser bezeichnet einen diagnostischen Fehler im Sinne eines kognitiven Bias. Er kann auftreten, wenn das abgebende Team einen bestimmten Aspekt überbetont und das aufnehmende Team dadurch beeinflusst wird [41].

Es ist davon auszugehen, dass durch die Anwendung einer Merkhilfe bei 
der Übergabe ein sog. „Framing-Effekt “ minimiert wird. Die Merkhilfe standardisiert und strukturiert die Übergabe, konsekutiv wird die Beeinflussungsmöglichkeit der Realitätswahrnehmung bei der adressierten Person reduziert. Darüber hinaus sollte das aufnehmende Team ausgesprochene Verdachtsdiagnosen immer kritisch hinterfragen, um nicht vorschnell zu einer Therapieentscheidung zu kommen. Das aktive Hinterfragen ist kein Zeichen mangelnder Wertschätzung, sondern dient lediglich der Vermeidung diagnostischer Fehler.

\section{Empfehlung 10}

\section{Am Ende der Übergabe soll dem auf- nehmenden Team die Möglichkeit von Fragen eingeräumt werden.}

Im Sinne einer Qualitätssicherungsmaßnahme sollte nach Abschluss der Notfallversorgung durch das aufnehmende Team eine Rückmeldung an die Besatzung des zuführenden Rettungsmittels erfolgen (Rettungsgesetz NRW $₫ 7 a$ Absatz 2). Hierbei ist das Strafgesetzbuch $\$ 203$ Verletzung von Privatgeheimnissen (,,ärztliche Schweigepflicht") zu beachten. Eine Rückmeldung zum Verlauf des Patienten ist nur an die Teammitglieder des Rettungsdiensts zulässig, die an der Behandlung des jeweiligen Patienten beteiligt waren (Behandlungszusammenhang).

\section{Empfehlung 11}

Dem zuführenden Team sollte aus Sicht des Qualitätsmanagements aktiv eine Rückmeldung zum Verlauf des Patienten gegeben werden.

\section{Organisatorische Voraussetzungen und Umsetzung}

Bis zum heutigen Zeitpunkt existiert in Deutschland weder auf Bundesebene noch auf Landesebene ein konsentiertes Vorgehen zur Übergabe in der zentralen Notaufnahme. Daher ist es gerade in der Notaufnahme zwischen Rettungsdienst und Klinik erforderlich, dass gemeinsame Sichtweisen („,shared understanding") existieren, die die Übergabe in einer strukturierten, teambasierten Weise bedienen. Ein standardisiertes Vorgehen schaltet durch die geregelten Abläufe und Inhalte potenzielle Fehlerquellen aus und erhöht die Patientensicherheit. Da viele potenzielle Fehler auf der Komplexität, der Unsicherheit, fehlendem Teammanagement bzw. Missverständnissen zwischen den Teammitgliedern in der Übergabesituation beruhen, sollten alle Beteiligten entsprechend qualitativ ausgebildet und trainiert werden.

\section{Empfehlung 12}

Teamwork, Kommunikation, Vertrauen, Situationsbewusstsein, Rollen und Verantwortlichkeiten sowie Konfliktlösung und Sicherheitskultur sollten in regelmäßigen Trainings mit allen Beteiligten, die eine Übergabe durchführen, geschult werden.

Übergaben sollten standardisiert und präzise sein. Sie sind zu jedem Zeitpunkt, an jedem Ort und für jeden Patienten in hoher Qualität durchzuführen. Um dieses Leistungsniveau zu erreichen, sind ein starkes Engagement, Ressourcen, ein Programm zur Längsschnittüberwachung sowie eine Verbesserung der Übergabepraktiken erforderlich. Letztlich dient dies dem Ziel, die besten Praktiken in die kulturellen Normen und Erwartungen aller Beteiligten zu integrieren.

Diese Aspekte sind von Starmer et al. mit der I-PASS-Studie eindrucksvoll belegt worden [42]. Im Rahmen der im New England Journal of Medicine publizierten Studie wurde ein "hand-off bundle“ für die mündliche Übergabe entwickelt; dieses enthält u.a. folgende Elemente: das „Mnemonic“, einen Workshop zu Teamwork, Kommunikation und Übergabetechniken; ein simuliertes Szenario zur praktischen Vertiefung des Workshops; ein E-Learning-Modul zur eigenständigen Erarbeitung von Lerninhalten; die professionelle Weiterentwicklung der Ausbildung; festgelegte Beobachtungskriterien für Bewertung und Feedback; eine Kampagne zur Nachhaltigkeit von Prozess- und Kulturentwicklung (mit Logo, Poster etc.). Die WHO empfiehlt ihren Mitgliedsländern den Einsatz ei- nes einheitlichen Vorgehens an allen Schnittstellen der Patientenübergabe.

Unabdingbar, wie von der JCAHO 2017 festgestellt, sind darüber hinaus die Schaffung eines geeigneten Arbeitsumfelds sowie die Vorhaltung eines adäquaten Equipments, ausreichendes Personal und vor allem, dass eine entsprechende Kultur in der Übergabe aktiv gelebt wird [10].

Eine nationale Initiative zur Standardisierung des Übergabeprozesses in der ZNA wäre für Deutschland, entsprechend den in England, Australien und USA bereits erfolgten Initiativen, dringend anzustreben.

\section{Empfehlung 13}

Die Implementierung einer standardisierten interprofessionellen Übergabe sollte zu einem nationalen gesundheitspolitischen Ziel erklärt werden.

Langfristig ist zu fordern, dass in Analogie zu der geforderten Ersteinschätzung in der ZNA auch die Einführung von strukturierten Übergabeprozessen in externen Audits überprüft werden muss. Notwendig erscheint auch die einheitliche Aufnahme des Themas Übergabe (Übergabeschema) als Schulungsinhalt in die Curricula der bewährten präklinischen und klinischen Kurskonzepte zur Versorgung von Schwerverletzten, Reanimationspatienten oder Kindern. Ebenfalls sollte das Thema Übergabe bspw. in die Ausbildung zum Rettungssanitäter/Notfallsanitäter sowie in die Weiterbildung „Klinische Akut- und Notfallmedizin“" und "Notfallmedizin“ sowie „Notfallpflege“ Einzug halten. Darüber hinaus sollte das Thema „strukturierte Übergabe" frei zugänglicher Bestandteil verschiedener Fort- und Weiterbildungen, aber auch regionaler Konzepte werden. Denkbar wäre auch eine spezifische "Übergabefortbildung", die verpflichtend für alle sein könnte, bevor sie in der ZNA arbeiten.

\section{Empfehlung 14}

Die Lehre einer standardisierten Übergabe soll in alle relevanten Berufsausbildungen integriert werden. 
Eine gute Übergabe ist kein Zufallsprodukt. Repetitive Trainingseinheiten zur Übergabe und somit eine entsprechende Übergabekultur sollten etabliert werden. Schulungsmaßnahmen zur Durchführung einer erfolgreichen Übergabe, sowohl aus der Sicht des Senders als auch des Empfängers, müssen standardisiert werden. Das Personal ist in die Ausbildung mit Methoden wie Echtzeitbeobachtung und Leistungsfeedback, Rollenspielen und Simulationen sowie selbstständigem Lernen einzubeziehen $[10,43,44]$.

\section{Empfehlung 15}

\section{Best-Practice-Beispiele bei Übergaben sollten angestrebt werden und die qua- litativ hochwertigen Übergaben sollten zu einer gesundheitspolitischen Priori- tät werden. Entsprechende finanzielle und personelle Ressourcen sollten be- reitgestellt werden.}

\section{Korrespondenzadresse}

\section{PD Dr. med. I. Gräff, DESA}

Abteilung für Klinische Akut- und Notfallmedizin, Universitätsklinikum Bonn Bonn, Deutschland ingo.graeff@ukbonn.de

\section{Einhaltung ethischer Richtlinien}

Interessenkonflikt. I. Gräff, M. Pin, P. Ehlers, M. Seidel, B. Hossfeld, M. Dietz-Wittstock, R. Rossi, A. Gries, A. Ramshorn-Zimmer, F. Reifferscheid, T. Reinhold, H. Band, K.-H. Kuhl, M.-K. König, J. Kasberger, R. Löb, R. Krings, S. Schäfer, I.-M. Wienen, R. Strametz, K. Wedler, C. Mach, D. Werner und S. Schacher geben an, dass kein Interessenkonflikt besteht.

Für diesen Beitrag wurden von den Autoren keine Studien an Menschen oder Tieren durchgeführt. Für die aufgeführten Studien gelten die jeweils dort angegebenen ethischen Richtlinien.

\section{Literatur}

1. Australian Medical Association Limited (2006) Safe handover:safe patients. https://ama.com.au/sites/ default/files/documents/Clinical_Handover_0. pdf.Zugegriffen: 16. Juni 2020

2. Australian Commission for Saefty and Quality in Health Care (2008) Guidance on clinical handover for clinicians and manager. https:// www.safetyandquality.gov.au/sites/default/
files/migrated/TASDept-Training-ManualGenMedNursing.pdf.Zugegriffen: 16. Juni 2020

3. Joint Commission Center for Transforming Healthcare Hand-off communications. https:// www.centerfortransforminghealthcare.org/ improvement-topics/hand-off-communications/. Zugegriffen: 16. Juni 2020

4. Evans SM, Murray A, Patrick I, Fitzgerald M, Smith S, Cameron P (2010) Clinical handover in the trauma setting: a qualitative study of paramedics and trauma team members. Qual Saf Health Care 19(6):e57. https://doi.org/10.1136/qshc.2009. 039073

5. Fernando K, Adshead N, Dev S, Fernando A (2013) Emergency department multiprofessional handover. Clin Teach 10(4):219-223. https://doi. org/10.1111/tct.12018

6. Najafi Kalyani M, Fereidouni Z, Sarvestani RS, Hadian Shirazi Z, Taghinezhad A (2017) Perspectives of patient handover among paramedics and emergency department members; a qualitative study. Emerg (Tehran) 5(1):e76

7. Vidyarthi AR, Arora V, Schnipper JL, Wall SD, Wachter RM (2006) Managing discontinuity in academic medical centers: strategies for a safe and effective resident sign-out. J Hosp Med 1(4):257-266. https://doi.org/10.1002/jhm.103

8. Owen C, Hemmings L, Brown T (2009) Lost in translation: maximizing handover effectiveness between paramedics and receiving staff in the emergency department. Emerg Med Australas 21(2):102-107. https://doi.org/10.1111/j.17426723.2009.01168.x

9. O'Rourke M (2007) The Australian Commission on Safety and Quality in Health Care agenda for improvement and implementation. https://www.achsm.org.au/Portals/15/ documents/publications/apjhm/02-02/Vol._2 no._2_2007_APJHM.pdf. Zugegriffen: 16. Juni 2020

10. Sentinel Event Alert (2017) Inadequate hand-off communication. https://www.jointcommission. org/-/media/tjc/documents/resources/patientsafety-topics/sentinel-event/sea_58_hand_off_ comms_9_6_17_final_(1).pdf. Zugegriffen: 16. Juni 2020

11. Rodziewicz TL, Hipskind JE (2020) Statpearls: medical error prevention. StatPearls, Treasure Island

12. Zinn C (1995) 14,000 preventable deaths in Australian hospitals. BMJ 310(6993):1487. https:// doi.org/10.1136/bmj.310.6993.1487

13. Scott LA, Brice JH, Baker CC, Shen P (2003) An analysis of paramedic verbal reports to physicians in the emergency department trauma room. PrehospEmerg Care 7(2):247-251.https://doi.org/ 10.1080/10903120390936888

14. Bogenstätter Y, Tschan F, Semmer NK, Spychiger M, Breuer M, Marsch S (2009) How accurate is information transmitted to medical professionals joining a medical emergency? A simulator study. Hum Factors 51(2):115-125. https://doi.org/10. 1177/0018720809336734

15. Evans SM, Murray A, Patrick l et al (2010) Assessing clinical handover between paramedics and the trauma team. Injury 41(5):460-464. https://doi. org/10.1016/j.injury.2009.07.065

16. Theobald CN, Choma NN, Ehrenfeld JM, Russ S, Kripalani S (2017) Effect of a handover tool on efficiency of care and mortality for Interhospital transfers. JHosp Med 12(1):23-28. https://doi.org/ 10.1002/jhm.2669

17. Benjamin MF, HargraveS, NetherK (2016) Using the Targeted Solutions Tool ${ }^{\circledR}$ to improve emergency department handoffs in a community hospital. Jt Comm J Qual Patient Saf 42(3):107-118. https:// doi.org/10.1016/s1553-7250(16)42013-1

18. Keebler JR, Lazzara EH, Patzer BS et al (2016) Metaanalyses of the effects of standardized handoff protocols on patient, provider, and organizational outcomes. Hum Factors 58(8):1187-1205. https:// doi.org/10.1177/0018720816672309

19. Gries A (2007) „Advanced trauma life support (ATLS)“ Ein Konzept für Europa oder doch nicht? Anaesthesist 56:1101-1102 (https:// www.springermedizin.de/advanced-trauma-lifesupport-atls/8003532)

20. World Health Organization (2007) Communication during patient hand-overs. https://www. who.int/patientsafety/solutions/patientsafety/ PS-Solution3.pdf?ua=1. Zugegriffen: 16 . Juni 2020

21. World Health Organization (2008) Global priorities for research in patient safety. https:// www.who.int/patientsafety/research/priorities/ global_priorities_patient_safety_research.pdf? ua $=1$.Zugegriffen: 16 . Juni 2020

22. Dönitz S, Vetter C (2007) Positionspapier zur Voranmeldung und Übergabevon Patienten an der Schnittstelle Rettungsdienst-Klinik.https://www. dbrd.de/images/Dokumente/POSITIONSPAPIER_ BERGABE_2007.pdf.Zugegriffen: 16.Juni 2020

23. von Dossow V, Zwißler B (2016) Empfehlung der DGAI zur strukturierten Patientenübergabe in der perioperativen Phase - Das SBAR-Konzept. Anasthesiol Intensivmed Notfallmed Schmerzthe 51(2):136-137. https://doi.org/10.1055/s-0042101190

24. Thies K-C, Deakin CD, Lott C et al (2014) The European trauma course-Trauma teaching goes European. Resuscitation 85(1):19-20. https://doi. org/10.1016/j.resuscitation.2013.06.027

25. Rossi R (2020) Konzepte für eine strukturierte Patientenübergabe. Notfall Rettungsmed 23(2):93-98. https://doi.org/10.1007/s10049019-0599-8

26. Bouillon B (2017) S3-Leitlinie Polytrauma/ Schwerstverletzten-Behandlung. https://www. awmf.org/uploads/tx_szleitlinien/012-019I_S3_ Polytrauma_Schwerverletzten-Behandlung_ 2017-08.pdf. Zugegriffen: 16. Juni 2020

27. Schacher S, Glien P, Kogej M, Gräff I (2019) Strukturierte Übergabeprozesse in der Notaufnahme. Notfall Rettungsmed 22(1):3-8. https://doi.org/ 10.1007/s10049-018-0478-8

28. Kicken W, van der KlinkM, Barach P, Boshuizen HPA (2012) Handover training: does one size fit all? The merits of mass customisation. BMJ Qual Saf 21(Suppl 1):i84-i88. https://doi.org/10.1136/ bmjqs-2012-001164

29. Gibson SC, Ham JJ, Apker J, Mallak LA, Johnson NA (2010) Communication, communication, communication: the art of the handoff. Ann Emerg Med 55(2):181-183. https://doi.org/10.1016/j. annemergmed.2009.10.009

30. Rall M, Langewand S (2016) Für bessere und sicherere Zusammenarbeit: Crew Resource Management (CRM) im Rettungsdienst. In: Neumayr A Baubin M, Schinnerl A (Hrsg) Risikomanagement in der prähospitalen Notfallmedizin. Springer, Berlin, Heidelberg, New York, S21-36

31. Shannon CE, Weaver W (1949) The mathematical theory of communication. University of Illinois Press, Baltimore (https://ebookcentral.proquest. com/lib/gbv/detail.action?doclD=4792736)

32. Weichert O, Lenz W, Lenssen U, Kemmeries G (2013) Optimierung der Patientenversorgung durch strukturierte Anmeldung von kritisch kranken Patienten über den Rettungsdienst. 


\section{In eigener Sache}

Notfall Rettungsmed 16(2):129-134. https://doi. org/10.1007/s10049-012-1651-0

33. Ryan S, Ward M, Vaughan D et al (2019) Do safety briefings improve patient safety in the acute hospital setting? A systematic review. J Adv Nurs 75(10):2085-2098. https://doi.org/10.1111/jan. 13984

34. DIVI (2020) MIND/Notarztprotokoll. https://www. divi.de/empfehlungen/qualitaetssicherungintensivmedizin/mind-notarztprotokoll. Zugegriffen: 16 . Juni 2020

35. Solet DJ, Norvell JM, Rutan GH, Frankel RM (2005) Lost in translation: challenges and opportunities in physician-to-physician communication during patient handoffs. Acad Med 80(12):1094-1099. https://doi.org/10.1097/00001888-20051200000005

36. Brewster DJ, Waxman BP (2018) Adding kindness at handover to improve our collegiality: the K-ISBAR tool. Med J Aust 209(11):482-483

37. Spering C, Roessler M, Kurlemann T et al (2018) Optimierte Ressourcenmobilisation und Versorgungsqualität Schwerstverletzter durch eine strukturierte Schockraumalarmierung. Unfallchirurg 121(11):893-900. https://doi.org/10.1007/ s00113-017-0447-6

38. Tijunelis MA, Fitzsullivan E, Henderson SO (2005) Noise in the ED. Am J Emerg Med 23(3):332-335. https://doi.org/10.1016/j.ajem.2005.02.037

39. Donchin Y, Seagull FJ (2002) The hostile environment of the intensive care unit. Curr Opin Crit Care 8(4):316-320. https://doi.org/10.1097/00075198 200208000-00008

40. Haynes AB, Weiser TG, Berry WR et al (2009) A surgical safety checklist to reduce morbidity and mortality in a global population. $\mathrm{N}$ Engl J Med 360(5):491-499. https://doi.org/10.1056/ NEJMsa0810119

41. Strametz R, Bayeff-Filloff M (2019) Risikomanagement in der Notaufnahme, 1. Aufl.

42. Starmer AJ, Landrigan CP (2015) Changes in medical errors with a handoff program. $\mathrm{N}$ Engl J Med 372(5):490-491. https://doi.org/10.1056/ NEJMc1414788

43. Natafgi N, Zhu X, Baloh J, Vellinga K, Vaughn $T_{\text {, }}$ Ward MM (2017) Critical access hospital use of TeamSTEPPS to implement shift-change handoff communication. J Nurs Care Qual 32(1):77-86. https://doi.org/10.1097/NCQ.0000000000000203

44. Farnan JM, Paro JAM, Rodriguez RM et al (2010) Hand-off education and evaluation: piloting the observed simulated hand-off experience (OSHE). J Gen Intern Med 25(2):129-134. https://doi.org/ 10.1007/s11606-009-1170-y

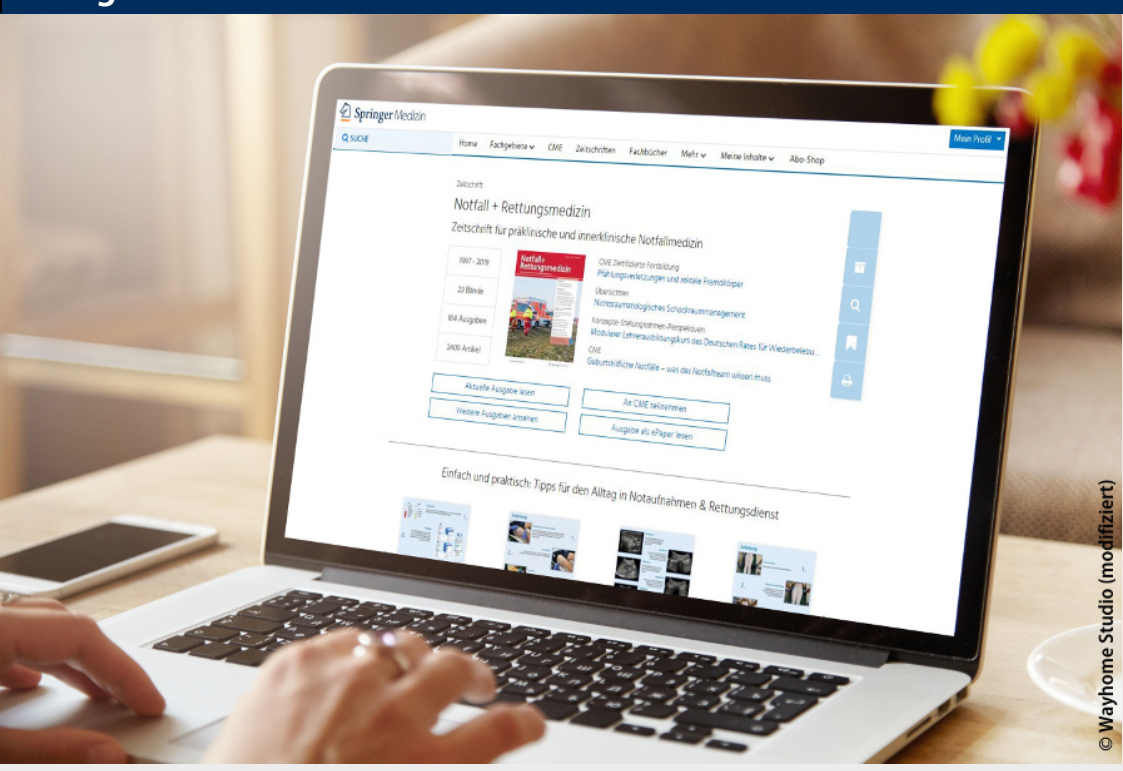

\section{Auch online Zugang zu allen Beiträgen von Notfall+Rettungsmedizin}

\section{Wussten Sie, dass Sie als Abonnent*in dieser Zeitschrift automatisch online}

Zugriffsrechte auf das gesamte Beitragsarchiv haben?

Durch Ihr Abonnement der Notfall+Rettungsmedizin erhalten Sie acht mal im Jahr die aktuelle Ausgabe der Zeitschrift nach Hause geliefert.

Doch damit nicht genug: Sie haben mit dem Abonnement außerdem Zugriff auf das gesamte Online-Archiv Ihrer Zeitschrift.

\section{Und so einfach geht es:}

Registrieren Sie sich einmal über www.springermedizin.de/register:

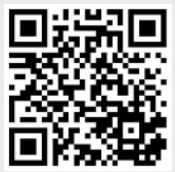

Über diesen QR-Code schnel und einfach registrieren

Bei der Registrierung geben Sie einfach Ihren Vor- und Nachname und Lieferadresse wie beim Abonnement der Zeitschrift (siehe Adressaufkleber auf Ihrem Heft) an. So kann im System die Zugehörigkeit zu Ihrer Zeitschrift sichergestellt werden.

Sollten Fragen oder Probleme auftauchen, wenden Sie sich einfach an den Kundenservice:

kundenservice@springermedizin.de
Aufgrund des Heilmittelwerbegesetzes dürfen die Inhalte der Website nur medizinischen Fachkreisen zur Verfügung gestellt werden. Bei der Anmeldung bitten wir Sie deshalb einen Berufsnachweis vorzulegen. Bei Mediziner*innen mit Mitgliedschaft in der deutschen Ärztekammer reicht die einheitliche Fortbildungsnummer (EFN). Als Angehörige* ${ }^{*}$ des nichtärztlichen Rettungsdienstfachpersonals oder Pflegepersonal schicken Sie eine Bestätigung der Arbeitsstelle. Sind Sie Student*in, dann bitte den Studiennachweis mit Angabe des Studiengangs ganz unkompliziert an kundenservice@springermedizin.de.

Mit Benutzername und Passwort haben Sie außerdem Zugang zu den freien Inhalten auf den Seiten von: https://www.springermedizin.de/ https://www.aerztezeitung.de/ 